\title{
Desempenho funcional de crianças com desnutrição crônica na faixa etária de um a três anos ${ }^{1}$
}

\author{
Monique Carla da Silva ${ }^{a}$, Ângela Cristina Dornelas da Silva ${ }^{b}$

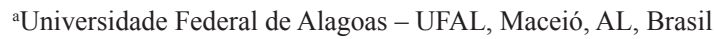 \\ bDepartamento de Terapia Ocupacional, Universidade Federal da Paraíba - UFPB, João Pessoa, PB, Brasil
}

\begin{abstract}
Resumo: A desnutrição infantil é um importante problema de saúde pública, sobretudo no Nordeste brasileiro, pois pode comprometer o crescimento e desenvolvimento das crianças de modo irreversível. Este estudo teve como objetivo avaliar o desenvolvimento funcional de crianças com desnutrição moderada ou grave na faixa etária de um a três anos de idade. Para tanto, foi realizado um estudo descritivo e com delineamento seccional com as crianças diagnosticadas com desnutrição crônica moderada ou grave que estavam ingressando em um centro de recuperação e educação nutricional na capital alagoana. Para mensurar o desempenho funcional das crianças foram utilizadas as escalas de autocuidado, mobilidade e função social da versão brasileira do Inventário de Avaliação Pediátrica de Incapacidade (PEDI). As condições sociodemográficas foram investigadas através de um questionário previamente elaborado para o estudo. Os resultados revelaram que três das 11 crianças avaliadas apresentaram desempenho funcional inferior ao esperado para sua idade. Das três crianças, uma apresentou atraso nas três áreas avaliadas, uma nas áreas de mobilidade e autocuidado e outra apenas na área de autocuidado. $\mathrm{O}$ autocuidado foi a área mais comprometida, sugerindo que o contexto socioeconômico familiar desfavorável interfere no engajamento das crianças nessa área de ocupação. Conclui-se que a desnutrição pode comprometer o desempenho em atividades cotidianas e que os programas de intervenção junto à criança desnutrida devem contemplar a estimulação ao desenvolvimento das atividades da vida diária ao mesmo tempo em que ocorre a reabilitação nutricional.
\end{abstract}

Palavras-chave: Desenvolvimento Infantil, Desnutrição, Atividades Cotidianas, Terapia Ocupacional.

\section{Functional performance of children with chronic malnutrition aged 1 to 3 years old}

\begin{abstract}
Child malnutrition is a major public health problem, especially in northeastern Brazil, because it can compromise the growth and development of children irreversibly. The aim of this study was to evaluate the functional development of children with moderate or severe malnutrition aged 1 to 3 years old. To this end, we conducted a descriptive study with cross-sectional design with children diagnosed with moderate or severe chronic malnutrition who were being assisted at a center for nutrition recovery and education in Joao Pessoa, Alagoas state, Brazil. The scales for self-care, mobility, and social function of the Brazilian version of the Inventory of Pediatric Evaluation of Disability (PEDI) were used to measure the functional performance of children. The sociodemographic conditions were investigated by a questionnaire previously developed for the study. The results showed that 3 of the 11 children evaluated presented functional performance lower than that expected for their age. Of these 3 children, one presented delay in the three areas assessed, one showed delay in the areas of mobility and self-care, and one only in the area of self-care. Self-care was the most affected area, suggesting that the unfavorable socioeconomic context of families affects the engagement of children in this area of occupation. We conclude that malnutrition can compromise performance in daily activities, and that intervention programs to malnourished children should include the stimulation of the development of activities of daily living together with nutritional rehabilitation.
\end{abstract}

Keywords: Child Development, Malnutrition, Activities of Daily Living, Occupational Therapy.

Autor para correspondência: Ângela Cristina Dornelas da Silva, Departamento de Terapia Ocupacional, Centro de Ciências da Saúde (CCS), Universidade Federal da Paraíba (UFPB), Campus I, Cidade Universitária, CEP 58051-900, João Pessoa, PB, Brasil, e-mail: angeladornelas@yahoo.com.br

Recebido em 10/3/2013; Revisão em 21/11/2013; Aceito em 21/12/2013. 


\section{Introdução}

O período atual está marcado por várias transições, dentre as quais se destaca a transição nutricional, na qual o número de desnutridos tem caído significativamente, ao mesmo tempo que o número de crianças e adultos obesos aumenta drasticamente. Em termos epidemiológicos, a prevalência da desnutrição no Brasil evoluiu de modo favorável entre as décadas de 1970 e 1990, com redução de 60\% dos casos (TONETE; CARVALHAES; TREZZA, 2002). Essa melhora esteve intimamente relacionada à ampliação de serviços de saneamento, saúde, educação e à oferta de programas de suplementação alimentar ao longo dessas três décadas (MONTEIRO, 1995).

A Pesquisa Nacional de Demografia e Saúde da Criança e da Mulher (PNDS) de 2006 revelou que a desnutrição no Brasil teve uma redução significativa da prevalência em relação a 1996, passando de 13,5\% para $6,8 \%$. Em relação à desnutrição crônica, que se traduz pelo déficit de altura para a idade, a regiáo Norte apresentou a maior prevalência, cerca de $14,8 \%$, entre as crianças até os cinco anos de idade. As regiōes Nordeste e Sudeste apresentaram uma prevalência de $5,7 \%$, a região Sul, $8,5 \%$ e a região Centro-Oeste, 5,6\% (BRASIL, 2008).

Entretanto, alguns estudos apontam para uma realidade mais desfavorável em estados e municípios da região Nordeste. Laurentino et al. (2006) encontraram uma prevalência de $12,1 \%$ de crianças menores de cinco anos de idade com déficit estatural em Pernambuco. Em Maceió, Alagoas, estudo realizado numa comunidade do "movimento dos sem teto" encontrou uma prevalência de 22,6\% de crianças com risco nutricional na faixa etária entre seis e 60 meses (FERREIRA et al., 2002).

Nessa perspectiva, a desnutrição infantil ainda se configura como um importante problema de saúde pública, pois além de afetar um número significativo de crianças, pode levar a consequências negativas irreversíveis para o crescimento e desenvolvimento das crianças (MONTE, 2000).

Por conceito, desnutrido é o indivíduo cujo organismo manifesta sinais clínicos provenientes da inadequação quantitativa (energia) ou qualitativa (nutrientes) da dieta ou decorrentes de doenças que determinem o mau aproveitamento biológico dos alimentos ingeridos (MONTEIRO, 1995). A ingestáo de uma dieta que seja inadequada de maneira qualitativa e/ou quantitativa é a causa da desnutrição primária, enquanto que a desnutrição secundária ocorre quando o indivíduo tem acesso ao alimento mas não o aproveita por causa de outras doenças, como anorexia e deficiência de absorção (FERREIRA, 2000).

$\mathrm{Na}$ criança, o termo desnutrição é sinônimo de falha no crescimento. As crianças desnutridas são mais baixas e/ou mais emagrecidas do que crianças sem desnutrição na mesma faixa etária (FUNDO..., 1998). O comprometimento do índice altura por idade, o chamado nanismo (stunting), indica que a desnutrição é crônica. O déficit de peso por altura, chamado emaciamento (wasting), indica que o comprometimento do estado nutricional é recente, caracterizando a desnutrição aguda (WORLD..., 1986).

De acordo com Ferreira (2000), o estado nutricional pode ser avaliado pela antropometria, que utiliza os índices peso para idade, peso para altura e altura para idade, comparados a uma curva padrão de referência. Assim ao utilizar o critério altura/ idade dessas curvas, pode-se constatar a ocorrência de desnutrição crônica na infância. É considerada desnutrição moderada quando a criança está dois desvios padrão abaixo da normalidade, ou seja, abaixo do valor esperado para seu sexo e idade. É considerada desnutrida grave a criança que apresentar três desvios padrão abaixo da normalidade.

A desnutrição incide de forma negativa sobre o desenvolvimento infantil, podendo alterar o desenvolvimento intelectual, pela interferência na saúde e nos níveis de energia necessários para o aprendizado, e retardar o crescimento físico (PLAZA et al., 2001). Mansur e Neto (2006) ressaltam que na falta de alimentação adequada nos primeiros anos de vida é possível que a criança não atinja seu verdadeiro potencial genético. Quando uma criança tem fome náo saciada, pode perder a motivação para explorar o ambiente e não adquirir certas habilidades cognitivas. Estudos também comprovaram que a baixa estatura, decorrente da desnutrição crônica na infância, também compromete a produtividade em tarefas na vida adulta (FLORÊNCIO et al., 2008).

As crianças desnutridas também são mais susceptíveis a prejuízos no desenvolvimento global porque comumente são oriundas de classe socioeconômica baixa, sofrem privaçáo cultural, educacional e afetiva, que causam graves déficits no desenvolvimento intelectual e emocional, os quais se potencializam quando sobrevém a desnutrição (CAMPOS et al., 1995). Eickmann et al. (2009) salientam que a pobreza repercute sobre o desenvolvimento porque as crianças de baixo nível socioeconômico são frequentemente expostas a múltiplos fatores adversos ao desenvolvimento. 
Nesse contexto, muitos estudos investigaram os efeitos da desnutrição no desenvolvimento infantil a partir de testes específicos para mensurar habilidades ou o desenvolvimento global. Entretanto, descrever o desenvolvimento em uma sequência de marcos sensoriais e motores, dentre outros, náo explica como as crianças aprendem a se engajar nas ocupaçóes (COELHO; REZENDE, 2007).

$\mathrm{Na}$ Terapia Ocupacional o termo ocupação é usado "para captar a dimensão e o significado da atividade do cotidiano" (CARLETO et al., 2010, p. 61). "O resultado de interaçóes entre a pessoa, o ambiente e a ocupação" é o Desempenho Ocupacional (LAW et al., 2009, p. 17), que pode ser investigado a partir de avaliaçôes que medem o desempenho funcional das pessoas.

Assim, este estudo teve como objetivo avaliar o desenvolvimento funcional de crianças com desnutriçáo crônica moderada ou grave na faixa etária de um a très anos de idade, partindo da compreensão de que tal investigação pode revelar aspectos importantes que devem ser considerados no planejamento de intervenções voltadas para a criança desnutrida.

\section{Procedimentos metodológicos}

Trata-se de um estudo descritivo, com delineamento transversal, realizado com crianças com desnutriçáo crônica moderada ou grave que estavam ingressando para tratamento no Centro de Recuperação e Educação Nutricional (CREN) de Maceió, Alagoas, em janeiro de 2010. Na ocasião, houve a admissão de 21 crianças para o sistema de semi-internato. Entretanto, 10 não fizeram parte da amostra. Três crianças foram excluídas para evitar vieses, uma vez que tinham condiçóes de saúde relacionadas diretamente com atraso no desenvolvimento global (prematuridade extrema e crises convulsivas frequentes), seis crianças não participaram da pesquisa porque as mães, por diversas razóes, tinham dificuldades de ir até o CREN e uma abandonou o tratamento logo no início. As comorbidades foram atestadas pelos pediatras do CREN.

Portanto, a amostra foi composta por 11 crianças na faixa etária de um a três anos de idade, diagnosticadas pela equipe de nutricionistas do CREN. O estado nutricional foi definido a partir de antropometria, utilizando-se os índices peso para idade, peso para altura e altura para idade, comparados ao padrão de referência proposto pelo National Center for Health Statistics (NCHS), utilizando-se o programa Epi Info. Para seleçáo de casos foi utilizado o critério altura para idade, considerando-se 2 DP (desnutriçáo moderada) ou 3 DP (desnutrição grave) abaixo da normalidade, por serem índices que identificam desnutrição crônica (FERREIRA, 2000).

O CREN é uma instituição sem fins lucrativos, mantida por uma organização não governamental denominada Nutrir (Associação de Combate a Desnutrição). Tem como objetivo combater a desnutrição primária em crianças com até seis anos de idade, oferecendo atendimento global e integrado a criança e sua família (FLORÊNCIO, 2003). Os desnutridos moderados e graves participam do semi-internato, passando o dia e recebendo assistência à saúde e educação. Os desnutridos leves são acompanhados no ambulatório.

Para avaliar o desempenho funcional das crianças foram utilizadas as escalas de autocuidado, mobilidade e função social da versão brasileira do Inventário de Avaliação Pediátrica de Incapacidade (PEDI). O PEDI é uma avaliação realizada através de entrevista estruturada com os pais ou responsáveis pela criança (MANCINI, 2005). Tem o propósito de ser uma avaliação clínica das capacidades funcionais e do desempenho típico em crianças jovens com inaptidōes, para descobrir déficits funcionais, monitorar progressos, ou avaliar o resultado de um programa terapêutico (HALEY et al., 1992). Esse teste foi traduzido para o português e adaptado para contemplar as especificidades socioculturais do Brasil, com permissão e colaboração dos autores da avaliação original (MANCINI; FIÚZA; REBELO, 2002).

A escala de autocuidado consiste na alimentação, no vestir e nas necessidades fisiológicas. A escala de mobilidade consiste em utilização de carro, cadeira, banheiro, andar em recinto fechado, ao ar livre e em escadaria. A escala de função social consiste em compreensão, fala, interações com amigos e na comunidade. A capacidade é medida pela identificação de habilidades funcionais para as quais a criança demonstrou domínio e competência (EMPELEN et al., 2005).

As condiçôes sociodemográficas foram investigadas através de um questionário previamente elaborado para o estudo. Para identificar a classe econômica das famílias foi utilizado o critério de Classificação Econômica Brasil da Associação Brasileira de Empresas de Pesquisa (ABEP). O critério foi desenvolvido para identificar a classe econômica de pessoas e famílias urbanas por meio do poder de compra e não para classificar a população em classes sociais. A classificação é feita através da pontuação atribuída à quantidade de certos aparelhos domésticos e automóveis que a família possui, somada 
à pontuação atribuída ao grau de escolaridade do chefe da família (ASSOCIAÇÃO..., 2008).

As entrevistas foram iniciadas após a assinatura do Termo de Consentimento Livre e Esclarecido pelas mães das crianças selecionadas para o estudo. $\mathrm{O}$ estudo obedeceu às normas exigidas pela resolução 196/96 do Conselho Nacional de Saúde e foi aprovado pelo Comitê de Ética em Pesquisa da Universidade Estadual de Ciências de Saúde de Alagoas - Uncisal em agosto de 2009.

A análise dos resultados se deu através da transformaçáo do escore bruto em escore padronizado normativo, de acordo com as tabelas do teste utilizadas para essa conversão, considerando a idade cronológica da criança.

O escore padronizado normativo informa sobre o desempenho esperado de crianças de mesma faixa etária, com desenvolvimento normal.

As crianças com os escores normativos entre $30 \mathrm{e}$ 70 são consideradas com desenvolvimento normal. Escores normativos inferiores a 30 ilustram atraso ou desempenho significativo inferior ao demonstrado por crianças da mesma faixa etária.

\section{Resultados}

A Tabela 1 apresenta os dados sociais e demográficos da amostra. Observa-se a baixa escolaridade materna, pois nenhuma mãe cursou o ensino médio e $45,5 \%$ delas têm até quatro anos de estudo, apenas. As residências, em sua maioria, abrigam muitos membros, tendo $63,6 \%$ delas cinco ou mais moradores. As 11 famílias estão inseridas entre as classes econômicas $\mathrm{C} 2$ e $\mathrm{D}$, sendo maioria pertencente à classe $\mathrm{D}(54,5 \%)$. A maior parte das crianças é do sexo feminino $(63,6 \%)$.

O questionário de classe econômica revelou também que três (27\%) chefes de família estudaram até o equivalente ao quarto ano do ensino fundamental, cinco $(45 \%)$ concluíram o quinto ano e apenas três (27\%) tinham o ensino fundamental completo.

Do total da amostra, quatro crianças (36\%) tinham irmáos desnutridos e sete $(64 \%)$ das 11 crianças estudadas tinham irmãos mais novos.

A Tabela 2 apresenta os resultados do PEDI. Ao analisar os resultados pelas escalas utilizadas percebe-se que o autocuidado foi o mais prejudicado, tendo três crianças apresentado desempenho inferior ao esperado para a idade. $\mathrm{Na}$ escala de mobilidade, o atraso pôde ser observado em duas crianças e na função social apenas uma teve desempenho inferior a outras crianças na mesma idade.

Ao analisar os resultados por criança observa-se que três crianças $(27,3 \%)$ apresentaram prejuízo no desempenho funcional. Uma criança apresentou prejuízo nas três escalas utilizadas, uma em duas escalas e uma em apenas uma escala.

\section{Discussão}

As características sociodemográficas das famílias configuram o estado de pobreza em que a população estudada vive. A escolaridade materna não ultrapassou os oito anos de estudo, assim como a escolaridade do chefe da família. O domicílio da maioria das famílias abriga mais de cinco moradores e tem mais de uma criança menor de três anos residindo no mesmo espaço. Parte das famílias tem mais de

Tabela 1. Características sociodemográficas de mães e suas crianças desnutridas, Maceió, 2010.

\begin{tabular}{llll}
\hline \multicolumn{2}{c}{ Variáveis sociodemográficas } & N & $\%$ \\
\hline Escolaridade da mãe & 0 a 4 anos de estudo & 5 & 45,5 \\
\multirow{3}{*}{ Moradores por domicílio } & 5 a 8 anos de estudo & 6 & 54,5 \\
\multirow{3}{*}{ Idade da criança } & até 4 moradores & 4 & 36,4 \\
& 5 ou mais moradores & 7 & 63,6 \\
\multirow{5}{*}{ Sexo da criança } & 1 ano & 2 & 18,1 \\
\multirow{3}{*}{ Classe econômica } & 2 anos & 4 & 36,4 \\
& 3 anos & 5 & 45,5 \\
& Feminino & 7 & 63,6 \\
& Masculino & 4 & 36,4 \\
& C2 & 2 & 18,2 \\
& D & 6 & 54,5 \\
\end{tabular}


Tabela 2. Desempenho funcional de crianças desnutridas, Maceió, 2010.

\begin{tabular}{cccc}
\hline \multirow{2}{*}{ Crianças } & \multicolumn{3}{c}{ Escores normativos $( \pm$ DP) } \\
\cline { 2 - 4 } & Autocuidado & Mobilidade & Função social \\
\hline 1 & $23,5( \pm 3,4 \mathrm{DP})$ & $<10$ & $34,1( \pm 3,2 \mathrm{DP})$ \\
2 & $27,7( \pm 3,2 \mathrm{DP})$ & $25,7( \pm 3,9 \mathrm{DP})$ & $20,1( \pm 3,8 \mathrm{DP})$ \\
3 & $44,5( \pm 2,8 \mathrm{DP})$ & $31,4( \pm 3,9 \mathrm{DP})$ & $35,0( \pm 2,8 \mathrm{DP})$ \\
4 & $57,2( \pm 2,4 \mathrm{DP})$ & $46,5( \pm 3,9 \mathrm{DP})$ & $50,5( \pm 3,1 \mathrm{DP})$ \\
5 & $65,6( \pm 2,9 \mathrm{DP})$ & $37,9( \pm 3,2 \mathrm{DP})$ & $54,4( \pm 3,0 \mathrm{DP})$ \\
6 & $64,0( \pm 3,6 \mathrm{DP})$ & $48,5( \pm 4,4 \mathrm{DP})$ & $53,3( \pm 2,7 \mathrm{DP})$ \\
7 & $51,1( \pm 3,2 \mathrm{DP})$ & $34,9( \pm 3,2 \mathrm{DP})$ & $47,7( \pm 3,2 \mathrm{DP})$ \\
8 & $28,9( \pm 3,1 \mathrm{DP})$ & $40,8( \pm 4,7 \mathrm{DP})$ & $41,3( \pm 3,1 \mathrm{DP})$ \\
9 & $41,4( \pm 2,8 \mathrm{DP})$ & $34,9( \pm 5,2 \mathrm{DP})$ & $33,7( \pm 2,5 \mathrm{DP})$ \\
10 & $65,5( \pm 2,9 \mathrm{DP})$ & $67,2( \pm 5,7 \mathrm{DP})$ & $60,8( \pm 3,0 \mathrm{DP})$ \\
11 & $49,3( \pm 3,2 \mathrm{DP})$ & $50,2( \pm 4,2 \mathrm{DP})$ & $34,1( \pm 3,2 \mathrm{DP})$ \\
\hline
\end{tabular}

uma criança com desnutrição e a maioria delas se insere nas classes econômicas D e E.

De acordo com o Critério Brasil de Classificação Econômica a classe econômica D corresponde a uma renda mensal em torno de $\mathrm{R} \$ 485,00$ e a classe $\mathrm{E}$ corresponde a uma renda mensal por volta de $\mathrm{R} \$$ 277,00. A minoria das famílias estudadas usufrui de uma renda em torno de $\mathrm{R} \$ 726,00$, que corresponde à classe C2 (ASSOCIAÇÃO..., 2008).

Um estudo caso-controle realizado no mesmo centro de recuperação nutricional apresentou como fatores de risco para a desnutriçáo crônica a baixa escolaridade materna ( $<4$ anos de estudo), a residência com mais de quatro moradores, a mãe ter mais de três filhos e, quando a família pertencia às classes econômicas $\mathrm{D}$ e E, o risco para desnutrição aumentava em 3,4 vezes em comparação com as famílias inseridas na classe C (SILVA, 2009).

Campino (1986) relata que os chefes de família com baixa escolaridade desempenham ocupaçóes cuja renda náo é suficiente para a provisão de uma boa alimentação e que a baixa escolaridade da mãe faz com que ela adote práticas em relação a nutrição e saúde de seus filhos com base em crenças que geralmente estão incorretas.

Compreendendo que os fatores de risco para o desenvolvimento de crianças de 0 a 6 anos podem ser: fatores socioeconômicos, fatores reprodutivos maternos, as condições da criança ao nascer, morbidade na infância, estrutura familiar, atenção a criança e os componentes maternos (FRANKENBURG et al., 1992), pode-se concluir que as crianças pesquisadas convivem com múltiplos fatores de risco para o desenvolvimento infantil e que podem comprometer a funcionalidade nas atividades cotidianas. As precárias condições das famílias, além de comprometerem o estado nutricional das crianças também inviabilizam a oferta de estímulos ambientais para o desenvolvimento das habilidades na infância.

Nesse sentido, Andrade et al. (2005) constataram que existe uma associaçáo entre a qualidade da estimulação no ambiente doméstico e o desempenho cognitivo infantil, verificando que a condição materna de trabalho e nível de escolaridade são fatores decisivos para uma boa estimulação ambiental. Os pesquisadores demonstraram que crianças que ocupam as primeiras ordens de nascimento e convivem com reduzido número de menores de cinco anos usufruem de melhor qualidade de estimulaçáo no ambiente doméstico.

Corroborando esta ideia, Martins et al. (2004) concluíram que a boa qualidade da criaçáo dos filhos requer gastos consideráveis em investimentos, por parte da família, que favoreçam o seu desenvolvimento e que a baixa renda familiar mensal, a baixa escolaridade materna, domicílios com mais de sete residentes e número de irmãos maior ou igual a quatro são fatores de risco para uma estimulação ambiental pobre.

Considerando que as crianças estudadas não apresentavam morbidades neurológicas, malformaçōes ou outras condiçóes de saúde relacionadas ao prejuízo no desempenho funcional, os resultados deste estudo sugerem que a desnutrição moderada ou grave também pode ser um quadro de saúde incapacitante, sobretudo porque é um agravo ligado à pobreza.

Sobre o assunto, Sawaya (2006) salienta que a fome é a necessidade básica de alimento que, quando não satisfeita, diminui a disponibilidade de qualquer 
ser humano para as atividades cotidianas e também para as atividades intelectuais. Consequentemente, a fome dificulta o engajamento da criança nas atividades cotidianas.

Neste estudo, o autocuidado foi a área de desempenho mais prejudicada. Humphry (2011) refere que as atividades de autocuidado são ocupaçóes determinadas pela cultura e por isso cheias de significado. $\mathrm{O}$ engajamento das crianças nessas atividades vai depender das situaçóes criadas para seu desenvolvimento e do significado que lhe é atribuído no meio familiar. É dentro do contexto familiar que a criança começa a desenvolver os padrôes de desempenho.

De acordo com Carleto (2010, p. 78):

[...] padrôes de desempenho se referem aos hábitos, rotinas, papéis e rituais usados no processo de envolvimento em ocupaçôes ou atividades [...] Papéis são conjuntos de comportamentos esperados pela sociedade, modelados pela cultura e que podem ser, além disso, conceituados e definidos pelo cliente. Os papéis podem fornecer orientação na seleção de ocupaçóes ou podem conduzir a padróes de envolvimento restritos e estereotipados.

No caso da extrema pobreza, dificilmente se estabelecem padróes de desempenho para garantir uma alimentação saudável. A presença do alimento e a frequência da alimentação são irregulares, comprometendo o estabelecimento de hábitos e rotinas relativas à alimentação. Tal situação também compromete o desenvolvimento de habilidades necessárias para o uso de utensílios adequados para cada tipo de refeição, habilidades para a higiene pessoal e utilização de vestuário adequado, dentre outras. A ausência desses padróes suprime o significado dessas ocupaçóes e a falta de significado faz com que essas ocupaçóes não façam parte do repertório das crianças (CLARK; WOOD; LARSON, 2002).

Gesell (1996) ressalta que os repertórios de crianças na faixa etária de um a três anos de idade são desenvolvidos gradativamente conforme vão sendo experimentadas e adquiridas habilidades necessárias para a independência nas atividades de alimentação, higiene, vestuário e sociabilidade.

$\mathrm{O}$ autor descreve que aos 18 meses a criança ensaia levar alimentos a boca com uso da colher e aos três anos ela já utiliza o utensílio com eficiência. Do mesmo modo que aos 18 meses a criança inicia o interesse pelas atividades de higiene e vestuário e aos três anos já é capaz de lavar partes do corpo, vestir e despir vários tipos de roupa. Em termos de sociabilidade, aos 18 meses a criança começa a se interessar pelos afazeres domésticos, gostando da companhia do adulto, ao passo que aos três anos consegue colaborar com tarefas domésticas, faz escolhas consistentes e prefere brincar com outras crianças (GESELL, 1996).

Por outro lado, os casos graves de desnutrição repercutem no desenvolvimento do cérebro (CHAVES, 1985) e no crescimento, refletindo negativamente no desempenho infantil. Crookston et al. (2010), em estudo longitudinal realizado no Peru, identificaram a baixa estatura em crianças de até cinco anos de idade como condição associada aos piores índices de desenvolvimento cognitivo.

Um estudo realizado por Miranda et al. (2007) comparou o perfil neuropsicológico de crianças eutróficas, desnutridas pregressas e crônicas e demonstrou que a desnutrição crônica foi relacionada ao prejuízo de linguagem expressiva e da memória operacional visuoespacial.

Consoante a Grieve e Gnanasekaran (2010, p. 65):

[...] a cognição compreende todos os processos mentais que nos permitem desenvolver atividades significativas na vida diária. Grande parte do nosso dia é passada em atividades habituais ou rotineiras [...] os hábitos não rotineiros precisam da prática para melhorar até que se tornem estabelecidos. As novas situações requerem planejamento e solução de problemas para se alcançar o objetivo desejado.

Nesse sentido, compreende-se a importância do desenvolvimento cognitivo para o desempenho ocupacional, revelando-se uma relação entre o estado de desnutrição e a falta de êxito nas tarefas de autocuidado, função social e mobilidade em crianças com desnutrição crônica. A falta de ânimo para explorar o ambiente soma-se ao pouco estímulo que o ambiente oferece, concorrendo para o mau desempenho das crianças.

Em relação ao instrumento utilizado para avaliar o desempenho funcional, foi observado que o PEDI se mostrou eficiente para investigar se as crianças dão conta dos desafios cotidianos, considerando seu estágio de desenvolvimento. Em soma, as informaçôes fornecidas pelo teste permite planejar açóes de modo mais objetivo para o enfrentamento dos prejuízos ao desenvolvimento infantil decorrentes da desnutrição. Espera-se, também, que ao aplicar o teste, a mãe ou responsável tome consciência das atividades que as crianças devem realizar no dia a dia e que, talvez, possa contribuir para mudanças de atitudes do adulto perante a criança. 


\section{Considerações finais}

O estudo demonstrou que crianças com desnutrição crônica devem ser submetidas a avaliaçôes funcionais, durante o processo de diagnóstico e tratamento, e não apenas a avaliaçóes clínicas e antropométricas. Nesse sentido, o PEDI pode ser útil na tomada de decisão no que se refere à atenção integral à saúde da criança com desnutrição.

Os resultados apontam para a necessidade de um acompanhamento multiprofissional, a fim de estimular o desenvolvimento de habilidades para que a criança possa responder às demandas do ambiente dentro de padrôes que promovam a saúde e o desenvolvimento pessoal.

Nessas perspectivas, o terapeuta ocupacional tem muito a contribuir com a equipe multiprofissional durante o processo de avaliação e acompanhamento dessa populaçáa, tendo em vista que é o profissional capacitado a promover o engajamento das crianças nas atividades significativas.

\section{Referências}

ASSOCIAÇÃO BRASILEIRA DE EMPRESAS DA PESQUISA - ABEP. Critério de Classificação Econômica Brasil. São Paulo: ABEP, 2008. Disponível em: <http:// www.abep.org>. Acesso em: 14 jun. 2010.

ANDRADE, A. S. et al. Ambiente familiar e desenvolvimento cognitivo infantil: uma abordagem epidemiológica. Revista de Saúde Pública, São Paulo, v. 39, n. 4, p. 606-11, 2005. http://dx.doi.org/10.1590/ S0034-89102005000400014

BRASIL. Ministério da Saúde. Pesquisa Nacional de Demografia e Saúde da Criança e da Mulher. Brasília: Ministério da Saúde, 2008. (Relatório).

CAMPINO, A. C. C. Aspectos sócio-econômicos da desnutrição no Brasil. Revista de Saúde Pública, São Paulo, v. 20 , n. 1, p. $83-101,1986$. http://dx.doi.org/10.1590/ S0034-89101986000100007

CAMPOS, A. L. R. et al. Aspectos nutricionais, psicológicos e sociais de mães de crianças desnutridas. Jornal de Pediatria, Rio de Janeiro, v. 71, n. 4, p. 214-218, 1995.

CARLETO, D. G. S. et al. Estrutura da prática da terapia ocupacional: domínio e processo. 2. ed. Revista Triângulo, Uberaba, v. 3. n. 2, p. 57-147, 2010.

CHAVES, N. Nutriçẫo e sistema nervoso. In: CHAVES, N. (Org.). Nutrição básica e aplicada. Rio de Janeiro: Guanabara Koogan, 1985. p. 207- 217.

CLARK, F.; WOOD, W.; LARSON, E. A. Ciência ocupacional da terapia ocupacional para o século XXI. In: NEISTADT, M. E.; CREPEAU, E. B. Willard \& Spackman: terapia ocupacional. 9. ed. Rio de Janeiro: Guanabara Koogan, 2002. p. 10-17.

COELHO, Z. A. C.; REZENDE, M. B. Atraso no desenvolvimento. In: CAVALCANTI, A.; GALVÃO,
C. Terapia ocupacional: fundamentação e prática. Rio de Janeiro: Guanabara Koogan, 2007. p. 299-307.

CROOKSTON, B. T. et al. Children who recover from early stunting and children who are not stunted demonstrate similar levels of cognition. The Journal of Nutrition, Rockville, v. 140, n. 11, p. 1996-2001, 2010. PMid:20844188. http://dx.doi.org/10.3945/jn.109.118927

EICKMANN, S. H. et al. Fatores associados ao desenvolvimento mental e motor de crianças de quatro creches públicas de Recife, Brasil. Revista Paulista de Pediatria, São Paulo, v. 27, n. 3, p. 282-288, 2009.

EMPELEN, R. V. et al. Epilepsy surgery does not harm motor performance of children and adolescents. Brain: A Journal of Neurology, Oxford, v. 128, p. 1536-1545, 2005.

FERREIRA, H. S. Desnutrição: magnitude, significado social e possibilidade de prevenção. Maceió: EdUFAL, 2000.

FERREIRA, H. S. et al. Saúde de populaçóes marginalizadas: desnutriçáo, anemia e enteroparasitoses em crianças de uma favela do "movimento dos sem teto", Maceió, Alagoas. Revista Brasileira de Saúde Materno Infantil, Recife, v. 2, n. 2, p. 177-185, 2002. http://dx.doi. org/10.1590/S1519-38292002000200010

FLORÊNCIO, T. T. et al. Short stature and food habits as determining factors for the low productivity of sugarcane labourers in the State of Alagoas, north-eastern Brazil. Archivos Latinoamericanos de Nutrición, Caracas, v. 58, n. 1, p. 33-39, 2008.

FLORÊNCIO, T. T. Projeto de Implantação do Centro de Recuperação e Educação Nutricional de Maceió - AL. Maceió: Nutrir Associação de Combate à Desnutrição, 2003.

FRANKENBURG, W. K. et al. DENVER II: training manual. 2nd ed. Denver: Denver Developmental Materials, 1992.

FUNDO DAS NAÇÓES UNIDAS PARA A INFÂNCIA - UNICEF. Situação mundial da infância 1998: a nutrição em foco. Brasília: UNICEF, 1998.

GESELL, A. A criança dos 0 aos 5 anos. 4. ed. São Paulo: Martins Fontes, 1996.

GRIEVE, J.; GNANASEKARAN, L. Neuropsicologia para terapeutas oucpacionais: cogniçáo no desempenho ocupacional. 3. ed. São Paulo: Santos, 2010.

HALEY, S. M. et al. Pediatric evaluation of disability inventory. Boston: New England Medical Center; PEDI Research Group, 1992.

HUMPHRY, R. Ocupação e desenvolvimento: uma perspectiva contextual. In: CREPAU, E. B.; COHN, E.; SCHELL, B. A. B. Willard \& Spackman: terapia ocupacional. 10. ed. Rio de Janeiro: Guanabara Koogan, 2011. p. 22-32.

LAURENTINO, G. E. et al. Déficit estatural em crianças em idade escolar e em menores de cinco anos: uma análise comparativa. Revista de Nutrição, Campinas, v. 19, n. 2, p. 157-167, 2006.

LAW, M. et al. Medida canadense de desempenho ocupacional. Belo Horizonte: UFMG, 2009. 
MANCINI, C. M. Inventário de Avaliação Pediátrica de Disfunção (PEDI): manual da versão brasileira adaptada. Belo Horizonte: UFMG, 2005.

MANCINI, M. C.; FIÚZA, P. M.; REBELO, J. M. Comparação do desempenho de atividades funcionais em crianças com desenvolvimento normal e crianças com paralisia cerebral. Arquivos de Neuro-Psiquiatria, São Paulo, v. 60, n. 2B, p. 446-452, 2002. http://dx.doi. org/10.1590/S0004-282X2002000300020

MANSUR, S. S.; NETO, F. R. Desenvolvimento neuropsicomotor de lactentes desnutridos. Revista Brasileira de Fisioterapia, São Carlos, v. 10, n. 2, p. 185-191, 2006.

MARTINS, M. F. D. et al. Qualidade do ambiente e fatores associados: um estudo em crianças de Pelotas, Rio Grande do Sul, Brasil. Cadernos de Saúde Pública, Rio de Janeiro, v. 20, n. 3, p. 710-718, 2004.

MIRANDA, M. C. et al. Neuropsychology and malnutrition: a study with 7 to 10 years-old children in a poor community. Revista Brasileira de Saude Materno Infantil, Recife, v. 7, n. 1, p. 45-54, 2007.

MONTE, C. M. G. Desnutrição: um desafio secular à nutrição infantil. Jornal de Pediatria, Rio de Janeiro, v. 76, p. 285-297, 2000. Suplemento 3.
MONTEIRO, C. A. A dimensão da pobreza, da fome e da desnutrição no Brasil. Estudos Avançados, São Paulo, v. 9, n. 24, p. 195-207, 1995.

PLAZA, B. et al. Algunas consideraciones sobre el impacto de la desnutricion en el desarrollo cerebral, inteligência y rendimento escolar. Archivos Latinoamericanos de Nutrición, Caracas, v. 51, n. 1, p. 64-71, 2001.

SAWAYA, S. M. Desnutrição e baixo rendimento escolar: contribuições críticas. Estudos Avançados, São Paulo, v. 20, n. 58, p. 133-146, 2006. http://dx.doi.org/10.1590/ S0103-40142006000300015

SILVA, A. C. D. Associação entre violência intrafamiliar e desnutrição infantil: um estudo caso-controle. 2009. $69 \mathrm{f}$. Dissertação (Mestrado em Ciências da Saúde)-Universidade Federal de Alagoas, Maceió, 2009.

TONETE, V. L. P.; CARVALHAES, M. A. B. L.; TREZZA, E. M. C. Evolução nutricional de crianças carentes atendidas por programa de suplementação suplementar. Pediatria, São Paulo, v. 25, n. 3, p. 101-109, 2002.

WORLD HEALTH ORGANIZATION - WHO. Use and interpretation of antropometrics indicators of nutricional status. Geneva: WHO, 1986. p. 929-941. (Bulletin of the World Health Organization, n. 64).

\section{Contribuição dos Autores}

Monique Carla da Silva: concepção do texto, organização das fontes de dados, redação do manuscrito. Ângela Cristina Dornelas da Silva: análise dos resultados, redaçáo e revisão do texto.

\section{Notas}

${ }^{1}$ Pesquisa desenvolvida para o trabalho de conclusão do Curso de Graduação em Terapia Ocupacional da Uncisal, aprovada pelo comitê de ética da mesma instituição. 